\title{
A computer tool for constructing and integrating inferences into text representations
}

\author{
FRANZ SCHMALHOFER \\ Interdisciplinary Center for Cognitive Science, University of Potsdam \\ Potsdam, Germany \\ and German Research Center for Artificial Intelligence, University of Kaiserslautern \\ Kaiserslautern, Germany \\ and \\ LUTZ FRANKEN and JÖRG SCHWERDTNER \\ German Research Center for Artificial Intelligence, University of Kaiserslautern \\ Kaiserslautern, Germany
}

\begin{abstract}
We present a new computer tool for modeling inference generation processes in text comprehension. With this tool, an explicit inference statement is constructed by marker passing in a joint text and knowledge base and a subsequent compilation process. In addition, the tool can be applied to describe knowl edge integration processes, which can produce spatial, causal, and other types of representations. Because the system provides explicit accounts for reproductive and creative inferencing, it exceeds the functionality of previous simulations in text research. Text researchers can employ this system as a common ground for comparing their different positions on inferencing in a more precise manner.
\end{abstract}

Within the last decade, inference generation has become one of the most central research topics in the area on text comprehension (Graesser, Singer, \& Trabasso, 1994; McKoon \& Ratcliff, 1992). Previous research on text comprehension has been greatly advanced by the development and application of computer simulation tools that have enabled researchers to more precisely specify the assumed cognitive representations and to subsequently simulate the postulated cognitive processes on a computer. For example, Turner (1987) has developed a computer tool, the propositional analysis system, which allows researchers to apply Kintsch's (1974) theory of language comprehension in a precise and more efficient manner. With this system, written information can be interactively transformed into a set of propositions that embody its meaning. Kieras (1991) has similarly developed a widget that parses simple texts into its propositions. Miller and Kintsch (1980) and Mross and Roberts (1990) have written computer programs that simulate how coherent text and situation representations are formed from propositions and other knowledge units. Such computer tools have been widely applied and have significantly

This research was supported by Grant Schm 648/1 1-1 to the first author from the Deutsche Forschungsgemeinschaft. This work was completed while the first author was a visiting professor at the Interdisciplinary Center for Cognitive Science at the University of Potsdam. The authors thank Ludger van Elst who has contributed invaluable ideas to this research. The authors would also like to thank Sarah Ransdell and an anonymous reviewer whose comments lead to many improvements in the readability of the paper. Correspondence should be addressed to F. Schmalhofer, German Research Center for Artificial Intelligence, Universität Kaiserslautern, Postfach 2080, D-67608 Kaiserslautern, Germany (e-mail: schmalho@dfki.uni-kl.de). sharpened our knowledge about human text comprehension processes (Britton \& Gülgöz, 1991; Goldman \& Varma, 1995; Schmalhofer \& McDaniel, 1996; Singer \& Halldorson, 1996).

For the more current research question of inference generation, on the other hand, no general computer tool has yet been developed that could model the construction of the different types of inferences as they have recently been described by Graesser et al. (1994). The computer tools that are available so far only address representational issues in combination with human memory processes. These tools are best applied for specifying the propositional structure that underlies a text and for specifying the way in which these propositions and other knowledge units are processed in human memory in the various processing cycles. When using these tools, one cannot specify some cognitive processes that, when applied, would then yield some explicit inference statement. A new computer tool is therefore required for modeling the generation of an inference statment and its subsequent integration into the text representation.

To support the modeling of inference construction processes, we have developed the EKI tool. EKI stands for Erzeugung kreativer Inferenzen, which can be loosely translated as Evolution of Creative Inferences. In addition to inference construction, the EKI tool can be used for performing goal-driven knowledge integration processes. These integration processes may be focused on a causal analysis (see Trabasso, van den Broek, \& Suh, 1989), the formation of spatial models (see Bower \& Morrow, 1990), or some other relational structure (e.g., some combination of spatial, causal, and motivational relationships). 
Graesser et al. (1994, p. 375) provided a classification of 13 different types of inferences. Some of these inference types may be modeled in a straightforward fashion. For example, their inference class 8 "instantiation of noun category" can be modeled simply by going down a subclass link in some well-structured knowledge base; one would thus instantiate "breakfast" to some more specific description, such as "bacon and eggs." Such inferences that require a lookup in the knowledge base and the subsequent identification of a subsumed concept (Brachman \& Schmolze, 1985) may be termed reproductive inferences. But there are also inference types that pose higher challenges to cognitive processing.

Some inference types will actually require that information from different representation spaces become related before the critical inference statement can be produced. For example, Graesser et al.'s inference class 5 concerns thematic inferences, and their inference class 13 concerns the derivation of the author's intent. Inferring an author's intent requires information about the text and information about the author. Because such inferences may be drawn only when different representation spaces are combined, their construction is computationally quite similar to creative inferences, as they have been described by Boden (1991).

A creative inference is an inference that does not simply (deductively or inductively) explicate some piece of information that is already implicitly contained in the available information; rather, it creates some unexpectedly novel statement by merging information from different representation spaces. A creative inference thus requires the immersion of information from another conceptual space, which is then used to construct the creative inference.

In this paper, we present the EKI system, which has been developed for simulating creative (e.g., thematic or author's intent) and reproductive (e.g., instantiation of noun category) inference generation processes within the framework of Kintsch's (1988) construction-integration theory, which assumes that text comprehension consists of a sequence of knowledge construction and knowledge integration episodes. This is also a central assumption of the EKI system. After describing the EKI system, we will show how this system may be applied to a particular text that has been widely studied with respect to inference generation (see McKoon \& Ratcliff, 1986).

\section{THE EKI SYSTEM}

With the EKI system, one can generate inferences by (1) connecting different knowledge spaces, (2) searching for paths between interesting points of the newly connected representation spaces, and (3) compiling these paths into explicit inference statements. Depending on the knowledge spaces that become connected and the particular specifications that guide the search for connecting paths, the resulting inferences may be quite novel and creative. More standard types of inferences (i.e., reproductive inferences), such as the instantiation of a noun category or the derivation of a causal antecedent, may also be simulated with the EKI system. For these types of inferences, it is usually sufficient to find a relevant path within a single knowledge base and then to select or compile the inference statement from this path. In addition, the EKI system can perform knowledge integration processes.

The EKI system consists of two major processing components - namely, the inferencing component and the integration component (see Figure 1). The inferencing component creates inferences from a knowledge net and inserts the created inferences into the knowledge net at proper locations. Because the knowledge net consists of a conglomerate of different knowledge segments and because the created inference may yield additional inconsistencies, the resulting knowledge net will usually contain many inconsistencies and redundancies. These diverse knowledge units, in combination with their latent organizations, are termed pluralistic views.

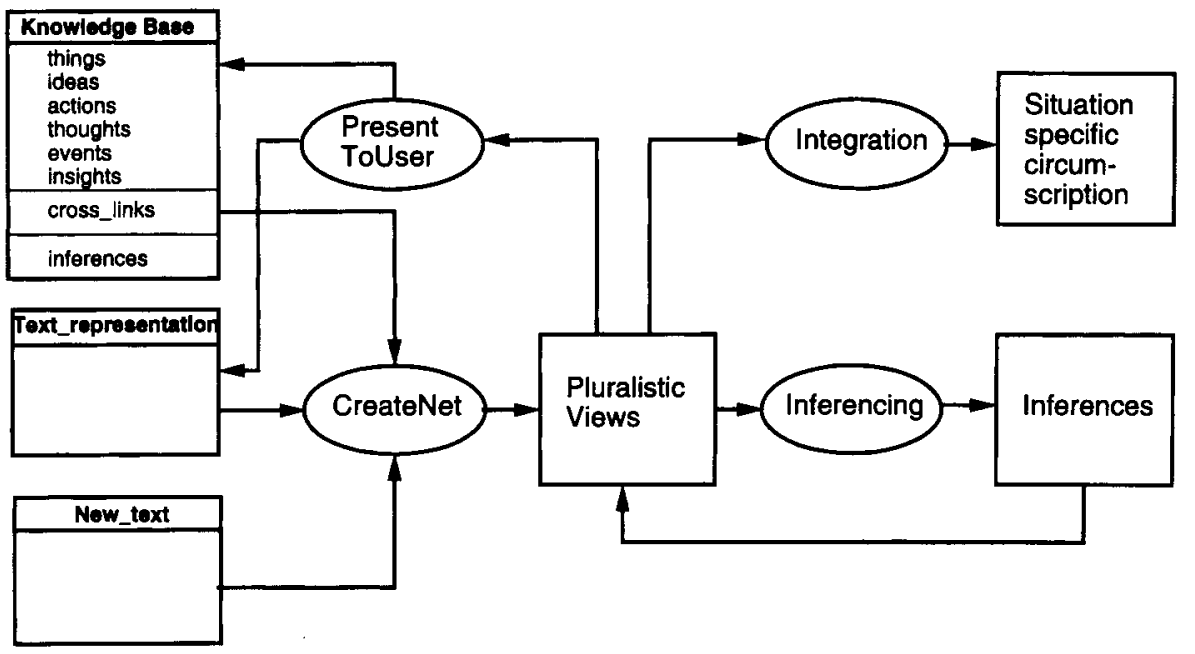

Figure 1. The architecture of the EKI system. 
The integration component searches the resulting knowledge net for coherence and consistency relations and thereby selects an appropriately chosen subnet so that some global coherence and consistency criteria are met. For instance, one may specify this integration process such that it would perform a causal analysis in the sense of Trabasso et al. (1989) or establish some spatial model (Bower \& Morrow, 1990). The resulting knowledge net, which may now represent a causal or spatial model, is called the situation-specific circumscription (see Figure 1). In addition to these two major components, the EKI system has a component for establishing the initial knowledge net (pluralistic views) from several well-structured knowledge bases and some text. Finally, there is a component for presenting the pluralistic views to the user.

The starting point for creating inferences is a number of separate knowledge bases, each of which is, in itself, well structured. For example, the things knowledge base consists of hierarchically structured object classes and respective subclass relationships. Each object is denoted by its name and several slots may be used for its description. The actions knowledge base similarly consists of hierarchically structured action classes. Because each of the different knowledge bases is a complete and correct representation of the respective segment of a domain, none of the knowledge bases affords creative inferences.

An affordance for creative inferences arises by associating or linking nodes either between or within some of the knowledge bases. The links that may introduce alternative readings to the previously precisely defined representations are called crosslinks. Relating the different knowledge bases by such crosslinks yields the possibility for discovering new relationships that were not already implicitly contained in the original knowledge bases. After such crosslinks have been inserted, the cohering knowledge base (pluralistic views) is obtained.

Creative inferences may now be constructed by focusing on two (or more) nodes, identifying relationships between these nodes, and then compiling the discovered relationship into some explicit inference statement. This explicit statement is subsequently inserted into the knowledge net. The creative inferences are, in themselves, additional crosslinks. The overall structure of the knowledge net is changed by these new links, and some additional arbitrariness may thereby be introduced. In other words, the good structure of the original knowledge bases is dissolved to a certain degree and thereby affords the development of some new structure.

The purpose of the integration process is to find such a new structure. This new structure is determined on the basis of some processing goal that identifies the nodes and types of links that are of central interest. By focusing on these nodes and links, a relatively coherent and consistent structure is then found (i.e., the situation-specific circumscription). The situation-specific circumscription, thus, is a novel structure that emerges from the orig- inal knowledge bases, the crosslinks (generated by the text), and the creative inferences that yield a shift of the focus in the knowledge net.

\section{System Architecture}

Figure 1 shows the architecture of the EKI system. On the left side, there are the various information sources that are to be provided by the user. The knowledge base, consisting of six segments (i.e., things, ideas, actions, thoughts, events, and insights), together with possible cross_links and the units of the text_representation, build the input to the system. Depending on the particular assumptions about human knowledge representations, a user (i.e., a text researcher) may specify some different knowledge base segments and a different number of segments. The new_text is entered into the system so that it corresponds unit by unit to the representational format of the text representation. There are two tools, which construct the initial pluralistic_views from the user's input (CreateNet) and present the result to the user (PresentToUser).

After this initial setup has been accomplished, inferences may be generated and a knowledge integration may be performed on the knowledge net (pluralistic_views). To generate a reproductive inference, the user must select a node in the net and then provide some specification that serves as the basis for finding a node that stands in a specific relationship to the selected node in the net. For example, when the node "breakfast" is selected in the net, the specification "subclass relation" would find the node "bacon and eggs." To explore creative inferences that may emerge from the knowledge net, the user must select at least two nodes and provide some specification that serves as the basis for finding relationships between these nodes. After such relationships have been discovered by the system in terms of connecting paths between the nodes (that satisfy the user-supplied specification), another specification determines how some explicit statement is compiled from these paths and inserted into the net. All of these component processes are depicted in Figure 1 by the procedure denoted as Inferencing.

To generate the situation-specific circumscription by the knowledge integration processes, the user identifies one focal node or several focal nodes of the net and provides a specification about which relationships are of interest and should therefore determine the situationspecific circumscription. For instance, for obtaining a causal model (see Trabasso et al., 1989), one may identify the setting and the outcome as the focal nodes and physical and motivational causes as the important relationships. The situation-specific circumscription (e.g., a causal model) is then calculated as the segment of the pluralistic views that satisfies the specified relationships. More specifically, the segment of the net consists of all the nodes that satisfy the given relationship with each of the focal nodes. These component processes are denoted in Figure 1 by the Integration procedure. In the following sections, the inferencing and integration procedures will be described in more detail. 


\section{Inferencing}

As can be seen in Figure 2A, the inference construction is performed in two steps. Possible relationships between two (or more) nodes are identified by a markerpassing procedure (Norvig, 1989). The resulting paths are then analyzed by a rule interpreter. The rules that are supplied to this interpreter specify under which conditions an inference may be constructed and the way in which an explicit inference statement is to be compiled out from some given path.

Marker-passing process. In order to find appropriate connections, the behavior of the marker can be programmed in terms of regular expressions. ${ }^{1}$ These regular expressions thus provide the specifications for which types of links can be traversed. A marker-passing process is started from each of the two nodes. For every collision point of the two markers, a connecting path is then determined. Depending on the particular specification that is used, the behavior of the markers may range from finding a very specific path over finding a concatenation of only transitive relations (e.g., some transitive closure) to allowing any arbitrary connections between the nodes.

Inference compilation. A rule interpreter is used for compiling explicit inference statements from a path and inserting these statements into the knowledge net. The rules for the rule interpreter consist of condition-action pairs. The condition part is again a regular expression that determines whether an explicit inference statement can be generated from a particular path by the action part of the rule. The action part then determines how the explicit inference statement is to be generated. Such an inference statement may consist of a single link that connects the two nodes from which the marker-passing process was started. Alternatively, the inference may consist of a new node and two links, where the two links connect the new node to each of the two nodes from which the markerpassing process was started.

\section{Integration Processes}

For performing the integration processes, the two or more nodes that are of focal interest must be identified by the user. Furthermore, for each of these focal nodes, a marker program must be supplied that determines the relations (links) that are most significant and should therefore guide the integration process. As can be seen in Figure $2 \mathrm{~B}$, the integration process is performed in two steps. In the first step, the marker-passing procedure is applied to flag all the nodes that are reachable from each given starting point by the respective marker program. In the second step, the situation-specific circumscription is determined by some integration criterion, which typically specifies that the situation-specific circumscription should be formed on the basis of the nodes that have been marked by all the different marker programs.

\section{EXAMPLE APPLICATION OF THE EKI SYSTEM}

The purpose of this section is to demonstrate how the EKI system can be used as a research tool for investigating inferencing and text comprehension in general. We are not proposing a new model, nor do we suggest any particular interpretation of an experimental data set. Instead, we will simply show how the EKI system can be employed as a tool for sharpening our theoretical understanding of human inference processes. For this purpose, consider a simple text that has been developed by McKoon and Ratcliff (1986) and that has been widely applied in studying inferencing: "The director and the cameraman were preparing to shoot close-ups of the actress on the edge of the roof of the 14-story building when suddenly the actress fell" (see text material 2A in the Appendix of McKoon $\&$ Ratcliff, 1986). From this text, a reader may possibly infer that the actress died from the fall.

\section{Method}

In a subject-paced experiment, we presented this text, phrase by phrase, to 10 participants, who were students at the University of Kaiserslautern. They were asked to verbalize all the relevant situational knowledge that came to their minds. Thus, we used the knowledge elicitation procedure that has been developed by Wetter and Schmalhofer (1988). Following their guidelines, we subsequently coded the elicited knowledge and represented

A

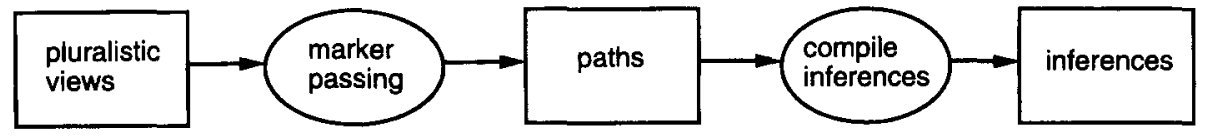

B

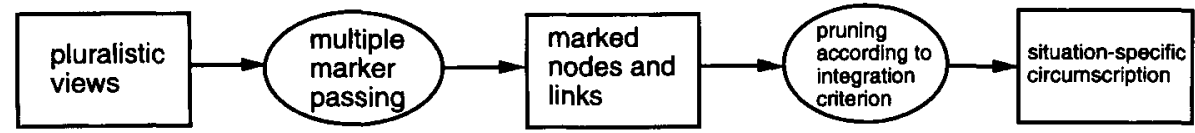

Figure 2. The components of (A) the inference generation process and (B) the knowledge integration process. 
it in the six segments of the EKI knowledge base. From the text and the knowledge base, we then created the pluralistic views with the CreateNet procedure (see Figure 1). Through the text, crosslinks were established among the various segments of the knowledge base. For instance, actress became linked to the class of human beings in the things knowledge base and to the class of professions in the ideas knowledge base. Because the knowledge base that we obtained from the verbal protocols did not contain a cause-effect relationship about falling from high heights causing death, the critical inference could not be produced by reproductive inference processes. Therefore, we explored whether the critical inference of the actress being dead may emerge from more creative inference processes.

For this purpose, we selected the nodes falling and actress as the focal nodes, and we supplied the marker program $[($ result $)+($ subclass action $) * \sim$ result $]$ to the falling node and the program $[(\sim$ subclass $) *($ action result $) *]$ to the actress node. The marker program of the falling node specifies that result links should be traversed, followed by subclass and action nodes, which, in turn, are followed by an inverse result link (i.e., an antecedent link). The marker program of the actress node, on the other hand, traverses superclass links (i.e., inverse subclass links), followed by action-result links.

\section{Results}

Figure 3 shows the paths that were identified by these marker programs. More specifically, via the nodes damage, death, dying, mortal, and human being, a connecting path was found between falling and actress. With the subsequent inference compilation process, the following two explicit inference statements were generated: (1) "The actress is performing the action of falling" and (2) "The result of falling is dying." In Figure 3, these inferences are represented by the two dashed lines that were added to the net by the inference compilation process. With such an explicit specification of inference construction, one may now, for example, sharpen the discussion of whether

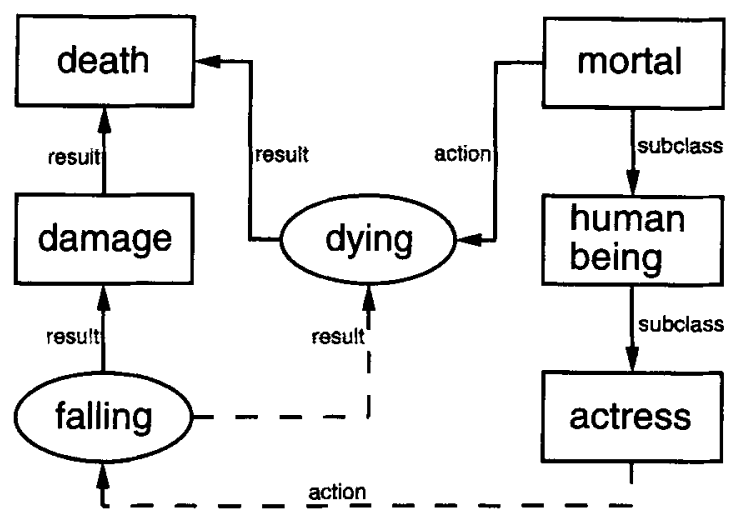

Figure 3. Example of an identified path and subsequent compilations of inference statements. From the path, the inference (actress-action $\rightarrow$ falling-result $\rightarrow$ dying) is constructed. priming effects in lexical decision or word-naming tasks may be caused by the spreading activation (marker-passing) process or the inference compilation process. Alternatively, the activated nodes and their marker programs could also be viewed as compound cues.

\section{IMPLEMENTATION}

The EKI system was designed in an object-oriented manner. The core components of the EKI system are the knowledge bases, the marker-passing process, and the inference compilation process. In the knowledge bases (knowledge net), one distinguishes nodes and links. All nodes are instances of object classes. There is one basic class, the BasicWorldClass, which has two subclasses: the StaticWorldClass representing static entities and the DynamicWuildClass representing changes. By using Java, we programmed a user interface, whose interaction style is very similar to that of a large number of other applications on the World-Wide Web. A demonstrator version of this system can be viewed over the Net. ${ }^{2}$ Because of the existing security restrictions of Java running under Netscape, the demonstrator cannot access any files. A regular version of the system, which allows full file access is also available. Since Java is an object-oriented and architecture-neutral programming language, the system will be highly portable to almost any hardware platform. Through the Java implementation, the EKI system can thus be demonstrated to more text researchers and subsequently shared by a larger number of users.

\section{CONCLUSION}

The EKI system differs from previously developed computer tools for text research (e.g., Mross \& Roberts, 1990; Turner, 1987), in that it allows one to explicitly model the inference generation process. Because the EKI system was developed within the framework of Kintsch's (1988) construction-integration theory, it does have some theoretical biases, but these biases are relatively weak. Therefore, it can be generally applied to different research questions. It can be used for simulating inference processes according to the specifications derived from different models, such as the validation model (Singer \& Halldorson, 1996) or a causal analysis model. The EKI system may indeed function as a general research tool for experimentalists and theorists, and it can provide a common ground for comparing different positions on inferencing in a more precise manner.

\section{REFERENCES}

Boden, M. A. (1991). The creative mind: Myths and mechanisms. New York: Basic Books.

Bower, G. H., \& Morrow, D. G. (1990). Mental models in narrative comprehension. Science, 247, 44-48.

Brachman, R. J., \& Schmolze, J. G. (1985). An overview of the KLONE knowledge representation system. Cognitive Science, 9, 171 216.

BritTon, B. K., \& GülGöz, S. (1991). Using Kintsch's computational model to improve instructional text: Effects of repairing inference 
calls on recall and cognitive structures. Journal of Educational Psychology, 83, 329-404.

GoldMAN, S. R., \& VARMA, S. (1995). CAPping the constructionintegration model of discourse comprehension. In C. A. Weaver III, S. Mannes, \& C. R. Fletcher (Eds.), Discourse comprehension: Essays in honor of Walter Kintsch (pp. 337-358). Hillsdale, NJ: Erlbaum.

Graesser, A. C., Singer, M., \& Trabasso, T. (1994). Constructing inferences during narrative text comprehension. Psychological Review, 101, 371-395.

KIERAS, D. (1991, January). A computer-based aid for comprehensible writing. Paper presented at the 2 nd annual winter text conference, Jackson Hole, WY.

KINTSCH, W. (1974). The representation of meaning in memory. Hillsdale, NJ: Erlbaum.

KINTSCH, W. (1988). The role of knowledge in discourse comprehension: A construction-integration model. Psychological Review, 95, 163-182.

McKoon, G., \& RATCLIFF, R. (1986). Inferences about predictable events. Journal of Experimental Psychology: Learning, Memory, \& Cognition, 12, 82-91.

McKoon, G., \& Ratcliff, R. (1992). Inference during reading. Psychological Review, 99, 440-466.

MiLleR, J. R., \& KINTSCH, W. (1980). Readability and recall of short prose passages: A theoretical analysis. Journal of Experimental Psychology: Human Learning \& Memory, 6, 335-354.

Mross, E. F., \& RoBerTs, J. O. (1990). The construction-integration model: A program and manual (Tech. Rep. No. 92-14). Boulder, $\mathrm{CO}$ University of Colorado, Institute of Cognitive Science.

Norvig, P. (1989). Marker passing as a weak method for text inferencing. Cognitive Science, 13, 569-620.
SChmalhofer, F., \& MCDANiEl, M. A. (1996). A unified model of forward and bridging inferences. Unpublished manuscript, Universität Kaiserslautern, DFKI, and University of New Mexico, Department of Psychology.

Singer, M., \& Halldorson, M. (1996). Constructing and validating motive bridging inferences. Cognitive Psychology, 30, 1-38.

Trabasso, T., VAN DEN BroEk, P., \& SuH, S. (1989). Logical necessity and transitivity of causal relations in stories. Discourse Processes, 12, 1-25.

TURNER, A. A. (1987). The propositional analysis system: Version 1.0 (Tech. Rep. No. 87-2). Boulder, CO: University of Colorado, Institute of Cognitive Science.

WeTter, T., \& SChMalhofER, F. (1988). Knowledge acquisition from text-based think-aloud protocols: Situational specifications for a legal expert system. In J. Boose, B. Gaines, \& M. Linster (Eds.), Proceedings of the European Knowledge Acquisition Workshop (EKAW '88) (pp. 331-345). Bonn: Gesellschaft für Mathematik und Datenverarbeitung.

\section{NOTES}

1. The program for matching regular expressions was written by Steven R. Brandt (http://www.win.nef/ stevesoft/pat/).

2. Additional information about EKI can be found at http://www. dfki.uni-kl.de/ schmalho/eki/eki.html.

(Manuscript received September 26, 1996; revision accepted for publication January 2, 1997.) 